\title{
ESTIMATES OF THE HARMONIC MEASURE OF A CONTINUUM IN THE UNIT DISK ${ }^{1}$
}

\author{
BY
}

CARL H. FITZGERALD, BURTON RODIN AND STEFAN E. WARSCHAWSKI

\begin{abstract}
The harmonic measure of a continuum in the unit disk is estimated from below in two ways. The first estimate is in terms of the angle subtended by the continuum as viewed from the origin. This result is a dual to the Milloux problem. The second estimate is in terms of the diameter of the continuum. This estimate was conjectured earlier as a strengthening of a theorem of D. Gaier. In preparation for the proofs several lemmas are developed. These lemmas describe some properties of the Riemann mapping function of a disk with radial incision onto a disk.
\end{abstract}

Let $E$ be a continuum in the closure of the unit disk $\Delta, 0 \notin E$. We are interested in lower estimates for the harmonic measure $\omega(0, E, \Delta)$, that is, the value at the origin of the bounded function which is harmonic in each component of $\Delta-E$ and which, roughly speaking, takes the value 1 at the boundary points belonging to $E$ and takes the value 0 on the rest of the boundary.

The classical Milloux problem (as in [1], for example) involves an estimate in terms of $E_{\text {rad }}$, the circular projection of $E$ onto a radius of $\Delta$, namely

$$
\omega(0, E, \Delta) \geqslant \omega\left(0, E_{\mathrm{rad}}, \Delta\right) .
$$

A natural conjecture, dual to the Milloux problem, would be

$$
\omega(0, E, \Delta) \geqslant \omega\left(0, E_{\text {circ }}, \Delta\right)
$$

where $E_{\text {circ }}$ is the radial projection of $E$ onto the circumference of $\Delta$. Theorem 1 of this paper states that the solution (2) of this dual Milloux problem is valid if $E_{\text {circ }}$ has length no greater than $\pi$.

Let $E_{\text {diam }}$ be an arc on the circumference of $\Delta$ which has the same diameter as $E$. Another natural conjecture is

$$
\omega(0, E, \Delta) \geqslant \omega\left(0, E_{\text {diam }}, \Delta\right) .
$$

(The conjecture (3) appears as Open Problem 3.21 in [2].) Theorem 2 of this paper states that (3) is valid. Simple examples show that either one of the estimates (2) or (3) may be stronger than the other depending on the particular continuum under consideration.

Estimates in terms of the projection $E_{\text {circ }}$ were studied by Maitland [10] for sets $E$ which are not necessarily connected. If Maitland's estimate is specialized to $z=0$

Received by the editors April 11, 1984.

1980 Mathematics Subject Classification. Primary 31A15, 30C85; Secondary 31A05, 30C20.

${ }^{1}$ This work was supported in part by the National Science Foundation.

(C1985 American Mathematical Society $0002-9947 / 85 \$ 1.00+\$ .25$ per page 
and $E$ a continuum, it becomes equivalent to an inequality which is inequality (2) with the left-hand side multiplied by a large constant (see Remark 1 at the end of §2). In Gaier [4] it is shown that Hall's lemma is valid with a constant of 1 if the set whose harmonic measure is to be estimated is an arc. Gaier's version of Hall's lemma can be applied in the disk to yield (2) in the special case that $1 \in E$ and $r i \in E$ for some $0<r \leqslant 1$. In general, Gaier's theorem and our Theorem 1 are not comparable.

Theorem 2 is a generalization of a theorem of Gaier [3, Theorem 9] which states that if $E$ is an arc with endpoints at 1 and $\zeta$ then

$$
\omega(0, E, \Delta) \geqslant(1 / \pi) \arcsin (|1-\zeta| / 2) .
$$

Our proof of Theorem 2 makes use of this theorem of Gaier.

A theorem of M. Lavrientiev [8, Theorem 8] states that if $E$ is an arc, if both endpoints of $E$ lie on $\partial \Delta$, and if $\operatorname{diam} E=d<1 / 2$, then $2 \pi \omega(0, E, \Delta)>d / 2$. Our Theorem 2 applied to this situation yields the stronger result $2 \pi \omega(0, E, \Delta) \geqslant$ $2 \arcsin (d / 2) \geqslant d$.

Other references related to Theorem 2 , in addition to those already mentioned, are Jenkins [6, 7] and Liao [9]. Jenkins [6] considers the same geometric situation as in [4] and obtains an estimate that takes account of the location of the free endpoint of the $\operatorname{arc} E$. [6, and 7] describe the arc which minimizes $\omega(0, E, \Delta)$ among all arcs $E$ in a given homotopy class with endpoints fixed.

1. Throughout this paper $\Delta$ denotes the unit disk $|z|<1$. For $0<r \leqslant 1, \Delta_{r}$ denotes the disk with an incision:

$$
\Delta_{r}=\Delta-\{z: r \leqslant z<1\} .
$$

The Riemann mapping function of $\Delta_{r}$ onto $\Delta$, extended continuously to the prime ends of $\Delta_{r}$ and normalized to send $0 \mapsto 0$ and $r \mapsto 1$, will be denoted by $F_{r}$. In the remainder of this section we abbreviate $F_{r}$ by $F$ since $r$ remains fixed.

This section is devoted to proving some mapping properties of $F$. Since $F$ is symmetric, $F(\bar{z})=\bar{F}(z)$, we state some of the properties for the upper half of $\Delta_{r}$ only.

LEMMA 1. Let $z=\rho e^{i \theta}$ with $0<\rho \leqslant 1$ and $0<\theta \leqslant \pi$. Then $F(z)=\rho_{1} e^{i \theta_{1}}$ with $\rho \leqslant \rho_{1}$ and $\theta \leqslant \theta_{1} \leqslant \pi$.

Proof. We first show that $\operatorname{Arg} F(z) \geqslant \operatorname{Arg} z$ for $z$ on the upper semicircle of $|z|=1$. Fix such a $z$ and let $\alpha=\left\{e^{i t}\right.$ : Arg $\left.z \leqslant t \leqslant \pi\right\}$. By monotoneity and conformal invariance $\omega(0, \alpha, \Delta) \geqslant \omega\left(0, \alpha, \Delta_{r}\right)=\omega(0, F(\alpha), \Delta)$ and consequently $F(\alpha)$ has length no greater than the length of $\alpha$ (Löwner's lemma). Since the endpoint -1 of $\alpha$ is left fixed by $F$ we conclude that $\operatorname{Arg} z \leqslant \operatorname{Arg} F(z) \leqslant \pi$.

Now consider the harmonic function $p(z)=\operatorname{Arg}(F(z) / z)$ in $\Delta_{r}$ where $\operatorname{Arg}(\cdot)$ is the branch with values in $(-\pi, \pi]$. We have proved that $p(z) \geqslant 0$ for $z$ on the upper semicircle. If $z$ is on the upper edge of the incision $[r, 1]$ then $p(z) \geqslant 0$ since, for such $z, \operatorname{Arg} z=0$ and $\operatorname{Arg} F(z) \geqslant 0$. 
By symmetry, $p(\bar{z})=-p(z)$. Thus $p(z)=0$ for $-1 \leqslant z \leqslant r$. The maximum principle gives $p(z) \geqslant 0$ in the upper half of $\Delta_{r}$, and from this we obtain the assertion $\theta \leqslant \theta_{1} \leqslant \pi$ of the lemma. The assertion $\rho \leqslant \rho_{1}$ is Schwarz's lemma.

LeMma 2. Let $z \in \Delta_{r}$ with $\operatorname{Re} z \leqslant r$. Then $|z-r| \leqslant|F(z)-1|$.

Proof. Consider the analytic function $g(z)=(z-r) /(F(z)-1)$ in $\Delta_{r} \cap\{\operatorname{Re} z$ $<r\}$. By Lemma 1 and symmetry $|z-1| \leqslant|F(z)-1|$ for $z$ on the circular part of the boundary of $\Delta_{r}$. Clearly $|z-r| \leqslant|z-1|$ for $\operatorname{Re} z \leqslant r$. Hence $|g(z)| \leqslant 1$ for $z$ on the circular part of the boundary of $\Delta_{r} \cap\{\operatorname{Re} z<r\}$.

Now consider points $z \in \Delta_{r}$ with $\operatorname{Re} z=r, \operatorname{Im} z>0$. Lemma 1 shows that either $\operatorname{Im} F(z)>\operatorname{Im} z$ or else $\operatorname{Re} F(z) \leqslant 0$. In the first case we have

$$
|z-r|=\operatorname{Im} z \leqslant \operatorname{Im} F(z) \leqslant|F(z)-1|,
$$

and in the second case

$$
|z-r| \leqslant 1 \leqslant \operatorname{Re}(1-F(z)) \leqslant|F(z)-1|, .
$$

Thus $|g(z)|<1$ on the vertical boundary of $\Delta_{r} \cap\{\operatorname{Re} z<r\}$.

To discuss $g(z)$ for $z$ near $r$, we consider $F\left(r+\zeta^{2}\right)$ for $\zeta$ in a small upper half disk centered at $\zeta=0$. By reflection, $F\left(r+\zeta^{2}\right)$ is analytic at $\zeta=0$ and $F\left(r+\zeta^{2}\right)=1+$ $i a_{1} \zeta+a_{2} \zeta^{2}+\cdots$ with $a_{1}>0$. Thus

$$
|g(z)|=\frac{|z-r|}{|F(z)-1|}=\frac{|z-r|^{1 / 2}}{\left|a_{1}+o(1)\right|} \rightarrow 0
$$

as $z \rightarrow r$. The maximum principle now yields $|g(z)| \leqslant 1$. in $\Delta_{r} \cap\{\operatorname{Re} z<r\}$ as desired.

The property of $F$ in the next lemma is presented for its own interest; it will not be needed for later results.

LEMMA 3. Fix $\theta$ with $0<\theta<\pi$. Then $\operatorname{Arg} F\left(\rho e^{i \theta}\right)$ is a nondecreasing function of $\rho$ for $0<\rho \leqslant 1$.

Proof. Since $\partial \operatorname{Arg} F(z) / \partial r=\operatorname{Im}\left[z F^{\prime}(z) /|z| F(z)\right]$, it suffices to show that $q(z)$ $=\operatorname{Im}\left[z F^{\prime}(z) / F(z)\right]$ is nonnegative for $z$ in the upper half of $\Delta_{r}$.

On the upper semicircle of $\Delta_{r}, \operatorname{Arg} F^{\prime}(z)=\operatorname{Arg}(F(z) / z)$; hence $q(z)=0$ there.

On the upper edge of the incision $(r, 1)$ we have $\operatorname{Arg} F^{\prime}(z)=\operatorname{Arg} F(z)+(\pi / 2)$. Therefore $q(z) \geqslant 0$ for these values of $z$.

For $-1 \leqslant z<r$ the values of $z, F(z)$ and $F^{\prime}(z)$ are real, and thus $q(z)=0$ here.

The local expansion of $F$ in terms of $\sqrt{z-r}$ in a small upper half disk about $r$ is bounded from below, although unbounded from above. Also near $z=1$, the local expansion of $F(z)$ shows $z F^{\prime}(z) / F(z)$ tends to zero as $z$ tends to 1 in $\Delta_{r}$. Thus $q(z) \geqslant 0$ in the upper half of $\Delta_{r}$ as desired.

2. We use the following notation. If $\Omega$ is an open set, $\alpha$ a closed set, and $z \in \Omega-\alpha$, then $\omega(z, \alpha, \Omega)$ denotes the value at $z$ of the bounded function which is harmonic in the component of $\Omega-\alpha$ containing $z$ and which, roughly speaking, takes the value 1 at boundary points belonging to $\alpha$ and the value 0 on the rest of the 
boundary. We say "roughly speaking" to avoid the problem of specifying where $\omega(z, \alpha, \Omega)$ actually takes on its prescribed boundary values.

THEOREM 1. Let $E$ be a continuum in $\{|z| \leqslant 1\}$ with $0 \notin E$. Let $E_{\text {circ }}=\{z /|z|$ : $z \in E\}$. If the length of $E_{\text {circ }}$ is no greater than $\pi$ then $\omega(0, E, \Delta) \geqslant \omega\left(0, E_{\text {circ }}, \Delta\right)$.

Proof. By rotating $E$ we may assume $E_{\text {circ }}=\left\{e^{i \theta}: 0 \leqslant \theta \leqslant \alpha\right\}$ where $\alpha \leqslant \pi$. Let $r$ be the point of $E \cap(0,1]$ having largest modulus. Recall the definition of the normalized Riemann map $F_{r}: \Delta_{r} \rightarrow \Delta$ (see (4)ff.) By monotoneity $\omega(0, E, \Delta) \geqslant$ $\omega\left(0, E, \Delta_{r}\right)$. Since only the tip of the slit belongs to $E$, it is not necessary to distinguish the sides of the slit to define $F_{r}(E)$. Therefore the principle of majorization [1, Theorem 3-1] applies to both $F_{r}$ and $F_{r}^{-1}$, hence

$$
\omega\left(0, E, \Delta_{r}\right)=\omega\left(0, F_{r}(E), \Delta\right) \text {. }
$$

That is, conformal invariance holds here. The continuum $F_{r}(E)$ contains the point 1 and has a projection onto $\partial \Delta$ of the form

$$
F_{r}(E)_{\text {circ }}=\left\{e^{i \theta}: 0 \leqslant \theta \leqslant \beta\right\},
$$

where, by Lemma $1, \beta$ satisfies $\alpha \leqslant \beta \leqslant \pi$.

For convenience we replace $F_{r}(E)$ by $E^{\prime}=\left\{\bar{z} e^{i \beta}: z \in F_{r}(E)\right\}$. The arc $E^{\prime}$ contains $e^{i \beta}$ and satisfies $E_{\text {circ }}^{\prime}=F_{r}(E)_{\text {circ }}$ and $\omega\left(0, E^{\prime}, \Delta\right)=\omega\left(0, F_{r}(E), \Delta\right)$.

Let $s$ be the point of $E^{\prime} \cap(0,1]$ having largest modulus. As before, we have

$$
\omega\left(0, E^{\prime}, \Delta\right) \geqslant \omega\left(0, E^{\prime}, \Delta_{s}\right)=\omega\left(0, F_{s}\left(E^{\prime}\right), \Delta\right) \text {. }
$$

The continuum $E^{*} \equiv F_{s}\left(E^{\prime}\right)$ has projection $E_{\text {circ }}^{*}=\left\{e^{i \theta}: 0 \leqslant \theta \leqslant \gamma\right\}$ where, by Lemma $1, \gamma$ satisfies $\beta \leqslant \gamma \leqslant \pi$. Since $1 \in E^{*}$ and $e^{i \gamma} \in E^{*}$ the monotoneity principle can be applied to yield $\omega\left(0, E^{*}, \Delta\right) \geqslant \omega\left(0, E_{\text {circ }}^{*}, \Delta\right)$.

We have seen that the length $\gamma$ of $E_{\text {circ }}^{*}$ is no less than the length $\alpha$ of $E_{\text {circ }}$. Hence $\omega\left(0, E_{\text {circ }}^{*}, \Delta\right) \geqslant \omega\left(0, E_{\text {circ }}, \Delta\right)$. The string of inequalities that has been obtained yields the desired conclusions $\omega(0, E, \Delta) \geqslant \omega\left(0, E_{\text {circ }}, \Delta\right)$.

REMARK 1 . Theorem 1 cannot be strengthened by allowing $E$ to consist of a finite union of continua. This assertion follows from the example of Hayman [5]. That example provides a set $E$ in the closure of the right half plane $H, E$ consisting of three arcs, and a superharmonic function $V(z)$ (in the notation of [5], $V(z)=$ $-u(z) / M)$ in $H$ which satisfies $V(z) \geqslant \omega(z, E, H)$ for $z \in H, V(x)<\frac{1}{2}$ for $x \in$ $(0, \infty)$. When transferred to $\Delta$ this gives a set $E^{\prime}$ of three arcs such that $\omega\left(0, E_{\text {circ }}^{\prime}, \Delta\right)$ $=\frac{1}{2}$ and $\omega\left(0, E^{\prime}, \Delta\right)<\frac{1}{2}$.

REMARK 2. Theorem 1 requires that the length of $E_{\text {circ }}$ be $\leqslant \pi$. Simple examples show that this upper bound $\pi$ cannot be replaced by $2 \pi-\varepsilon$. It would be of interest to know the sharp upper bound.

3. Let $d$ be the diameter of a continuum $E \subset \mathrm{Cl} \Delta$. Let $E_{\text {diam }}$ be a circular arc on $\partial \Delta$ which has the same diameter $d$. Then $\omega\left(0, E_{\text {diam }}, \Delta\right)=(1 / \pi) \arcsin (d / 2)$.

THEOREM 2. Let $E$ be a continuum contained in the closure of the unit disk $\Delta$. Let $d$ be the diameter of $E$. Then

$$
\omega(0, E, \Delta) \geqslant \frac{1}{\pi} \arcsin \frac{d}{2}
$$


Proof. Let $\zeta_{1}, \zeta_{2}$ be points of $E$ such that the diameter of $E$ is $\left|\zeta_{2}-\zeta_{1}\right|$. We may assume that $\left|\zeta_{2}\right| \leqslant\left|\zeta_{1}\right|$ and that $\zeta_{1}=r>0$. It follows that $E$ contains no points of the incision $[r, 1]$ other than $r$; hence $F_{r}(E)$ is a continuum in $\mathrm{Cl} \Delta$.

We have

$$
\omega(0, E, \Delta) \geqslant \omega\left(0, E, \Delta_{r}\right)=\omega\left(0, F_{r}(E), \Delta\right)
$$

The continuum $F_{r}(E)$ contains 1 and $F_{r}\left(\zeta_{2}\right)$. If we apply Gaier's theorem [3, Theorem 9] and then Lemma 2 we obtain

$$
\omega\left(0, F_{r}(E), \Delta\right) \geqslant \frac{1}{\pi} \arcsin \frac{\left|F_{r}\left(\zeta_{2}\right)-1\right|}{2} \geqslant \frac{1}{\pi} \arcsin \frac{\left|\zeta_{2}-r\right|}{2} .
$$

(We have applied Gaier's theorem to a continuum rather than a Jordan arc; Gaier's proof can be modified to make this legitimate.) The inequalities (5) and (6) yield the desired conclusion since $\left|\zeta_{2}-r\right|=d$ is the diameter of $E$.

\section{REFERENCES}

1. L. V. Ahlfors, Conformal invariants: topics in geometric function theory, McGraw-Hill, New York, 1973.

2. K. F. Barth, D. A. Brannan and W. K. Hayman, Research problems in complex analysis, Bull. London Math. Soc. 16 (1984), 490-517.

3. D. Gaier, Estimates of conformal mapping near the boundary, Indiana Univ. Math. J. 21 (1972), $581-595$.

4. _ A note on Hall's Lemma, Proc. Amer. Math. Soc. 37 (1973), 97-99.

5. W. K. Hayman, On a theorem of Tord Hall, Duke Math. J. 41 (1974), 25-26.

6. J. A. Jenkins, On a lemma of Tord Hall, Bull. Inst. Math. Acad. Sinica 2 (1979), 371-375.

7. On a problem concerning harmonic measure, Math. Z. 135 (1974), 279-283.

8. M. Lavrientiev, On the theory of conformal transformations Trudy Mat. Inst. Steklov. 5 (1934), 159-245. (Russian)

9. L. Liao, Certain extremal problems concerning module and harmonic measure, J. Anal. Math. 40 (1981), 1-42.

10. B. J. Maitland, A note on functions regular and bounded in the unit circle and small at a set of points near the circumference of the circle, Proc. Cambridge Philos. Soc. 35 (1939), 382-388.

Department of Mathematics, University of California, San Diego, la Jolla, California 92093 